\title{
PROGRESS TOWARDS THE PRACTICAL IMPLEMENTATION OF THE INTERMEDIATE BAND SOLAR CELL
}

\author{
A.Luque , A.Marti , P.Wahnón , L.Cuadra , C.Tablerc , C.Stanley, A.Mckee , D.Zhou , \\ R.Kónenkamp ; R.Bayón, A.Belaidí , J.Alonso , J.Ruiz , J.Fernández ,P.Palacios , N.López
}

\begin{abstract}
The intermediate band solar cell is a novel solar cell with the potential of achieving a limiting efficiency of 63.2 $\%$ on the basis of the absorption of two sub-bandgap photons to create one electron-hole pair. The path towards its practical implementation has started following three strategies: a) Engineering the IB material through quantum dot technology b) Direct synthesis of the IB material and c) creation of a localized absorber layer within a highly porous large bandgap semiconductor.
\end{abstract}

\section{INTRODUCTION}

The intermediate band (IB) solar cell is a novel solar cell with the potential of achieving an efficiency of $63.2 \%$ (detailed balance limit) under full concentration. Its operation is based on a semiconductor-like material that, thanks to the existence of an intermediate electronic band located within what in ordinary semiconductors constitutes the bandgap, is capable of absorbing two below bandgap photons to generate one extra electron-hole pair (Fig. 1). This band should be half-filted with electrons in order to supply both empty states for electrons pumped from the valence band (NB) and electrons to be pumped to the conduction band (CB). Due to this characteristic we also often describe this band as "metallic".

The IB solar cell (IBSC) bases its superior potential over single gap conventional solar cells (which efficiency is limited to $40.7 \%$ under the same operating conditions [1]) in its capability for increasing the photogenerated current without degrading its open-circuit voltage. Current is increased because sub-bandgap photons, which in a conventional single-gap solar cell are lost, are now absorbed and converted into electrical current. Open-circuit voltage is not degraded because, thanks to that the intermediate band material is sandwiched between two ordinary high bandgap semiconductors (Fig. 2), $p$ and $n$ doped, the output voltage is determined by the split of quasi-Fermi levels of electrons in the conduction band, $E_{F C}$, and holes, $E_{F V_{1}}$ in the valence band. This split is limited by the total bandgap $\varepsilon_{G}$ of the IB material rather than by the subbandgaps $a$ and $\varepsilon_{H}$. Having already been established its basic principles of operation [ $2-5]$, the path towards its practical implementation has started following three strategies: a) Engineering the intermediate band material through quantum dot technology, b) Direct synthesis of the intermediate band material and c) Creation of a localized absorber layer within a highly porous large bandgap semiconductor. The description of these strategies and the progress done is the objective of this paper. A brief description of each of them is given in the next paragraphs.

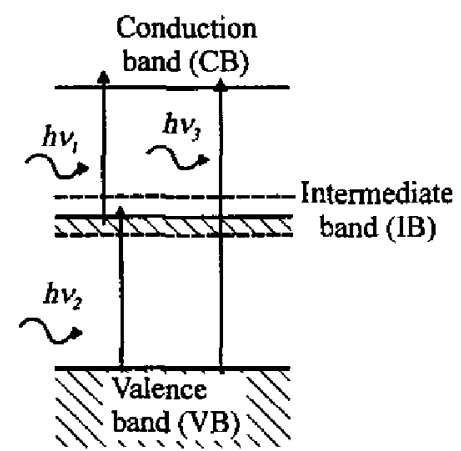

Fig. 1. Band structure of the metallic intermediate band material showing schematically the photon absorption processes involved.

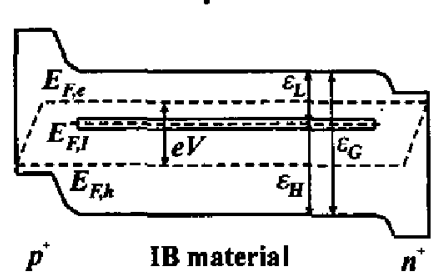

Fig. 2. Metallic intermediate band material sandwiched between two ordinary semiconductors. This provides the basic structure of the IBSC allowing the voltage, given by the quasi-Fermi level split $\left(E_{F, e}-E_{F, h}\right) / e$ to be limited by the total bandgap $\varepsilon_{G}$. 


\section{ENGINEERING THE INTERMEDIATE BAND MATERIAL THROUGH QUANTUM DOT TECHNOLOGY}

Fig. 3 illustrates the IBSC engineered through quantum dot technology. This approach is conceived at present more as a scieritific tool for demonstrating the operating principles of the IBSC rather than as a path towards its industrial implementation. Within this approach, the IB would arise from the states of the confined electrons in the dots. Candidate materials for this approach are Iny $\mathrm{Ga}_{1-x} A$ s for the dot material and $A l_{x} \mathrm{Ga}_{1-x} A A_{s}$ for the barrier material. The Stransky-Krastanow [6] growth mode appears as a suitable technology capable of providing a defect free high density of dots $\left(10^{17} \mathrm{~cm}^{-3}\right)$. Dots (D-D structures) and no other structures of higher dimensionality like wells (2-D) or wires (1-D) must be used because a true 0 -density of states must exist between the energy level of the confined electrons in the dots and the conduction and valence bands. Details of the theory behind the implementation of the IBSC by means of QDs can be found somewhere else, as for example, in Refs. [7. 10]

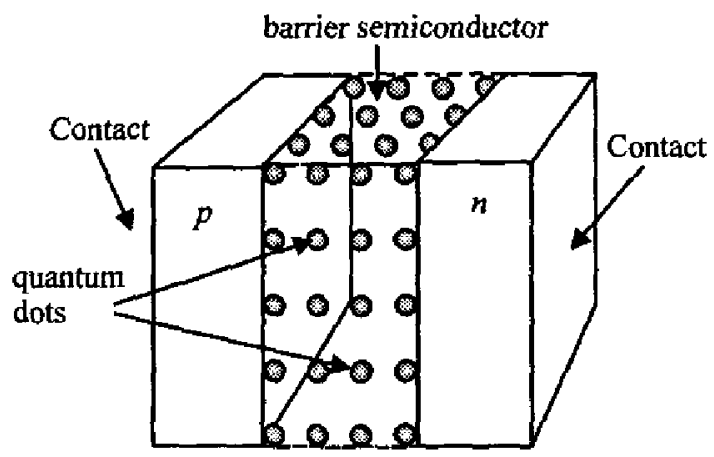

Fig. 3. Basic structure of the QD-IBSC.

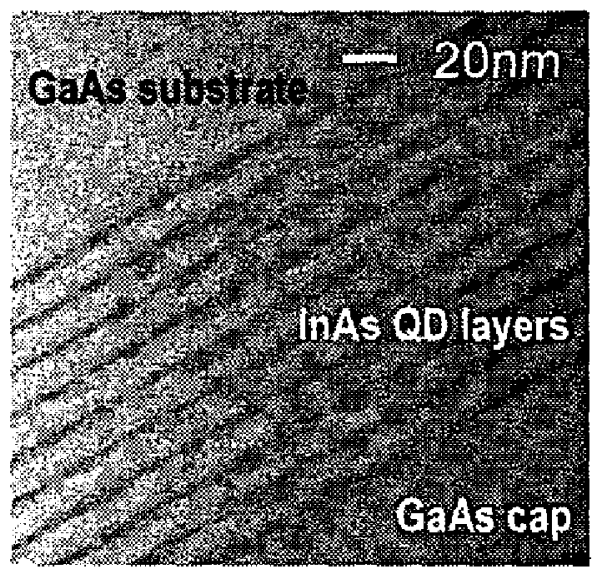

Fig. 4. TEM picture of 10 InAs $Q D$ layers embedded in GaAs for the QD-IBSC. (Coutesy of Dr. Richard Langford, University of Oxford).
Prototypes of this structure are being manufactured at the University of Glasgow and at Compound Semiconductor Technologies. At present, prior to the growth of a complete cell structure, experiments are focusing on the reliability, reproducibility and quality of the array of dots that can be manufactured. For the first trials, InAs has been selected as the material for manufacturing the dots and GaAs for the barrier.

At this respect, Fig. 4 shows a TEM picture of the cross section from one of the test samples. The volumetric density of dots is about $5 \times 10^{15} \mathrm{~cm}^{-3}$ and is achieved through a surface density of dots of $2 \times 10^{10} \mathrm{~cm}^{-2}$ per layer. Layers are spaced about $20 \mathrm{~nm}$. The base of the pyramidal dots typically sizes about $20 \mathrm{~nm}$. According to the model described in [9], this density of dots should suffice to clamp the quasi-Fermi level of the IB within $0.3 \mathrm{kT}$ up to operation at 100 suns if the density of states in the $C B$ and $V B$ is below $10^{18} \mathrm{~cm}^{-3}$.

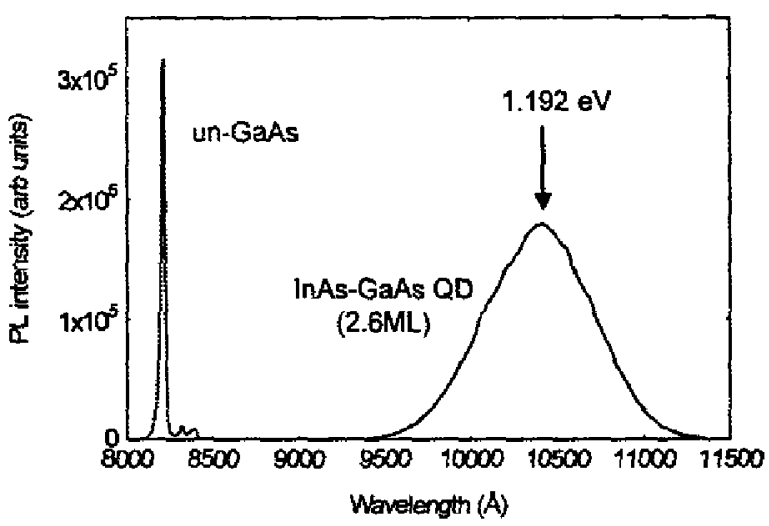

Fig. 5. Photoluminescence signal (plot at the right) from a single layer of InAs-GaAs QDs at $9 \mathrm{~K}$. The signal of an un-doped GaAs sample is also included for comparison.

(1).

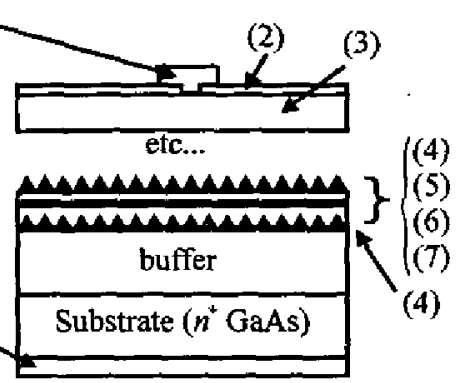

Fig. 6. Detail of the cross section of a QD-IBSC that includes $\delta$-doping layer to supply electrons for half-filling the IB: (1) Metal contacts (2) Window and antireflecting layers (3) p GaAs-emitter (4) InAs dots layer (5) GaAs layer (6) n type $\delta$-doping layer (7) GaAs layer.

Photoluminescence measurements (Fig. 5), have been carried out over samples consisting of one single layer of dots. Measurements peak at $1.2 \mathrm{eV}$ suggesting. to the IBSC theory is concerned, that we would be working with a system characterized by $a \approx 0,2 \mathrm{eV}, a+1,2 \mathrm{eV}$ 
and $\varepsilon \sigma=1,4 \mathrm{eV}$. For reference we shall mention that the potential efficiency of this system, according to the fundamental IBSC theory [2] would be $40.3 \%$ at 100 suns to be compared with the $53.6 \%$ of the optimized device ( $\sigma_{L}=0.82 \mathrm{eV}$ and $s_{1}=1.38 \mathrm{eV}$ ). In addition, the strength of the photoluminescence signal obtained also suggests a reasonable material quality.

In order to half-fill the IB with electrons, future steps aim to the introduction of $n$-type $\delta$-doping layers inbetween each two layers of QDs according to the schematics shown in Fig. 6 .

\section{DIRECT SYNTHESIS OF THE INTERMEDIATE BAND MATERIAL}

This approach pursues the direct synthesis of the intermediate band material with the long-term goal of reducing costs. At this respect, energy band diagrams of different alloys are being routinely calculated at the Instituto de Energia Solar. It has been found, for example, that the bandgap diagram of the compound GaAsTi, with the Ti properfy substituting some As atoms in the original GaAs crystal lattice, should exhibit such intermediate band as shown in Fig. 7 .

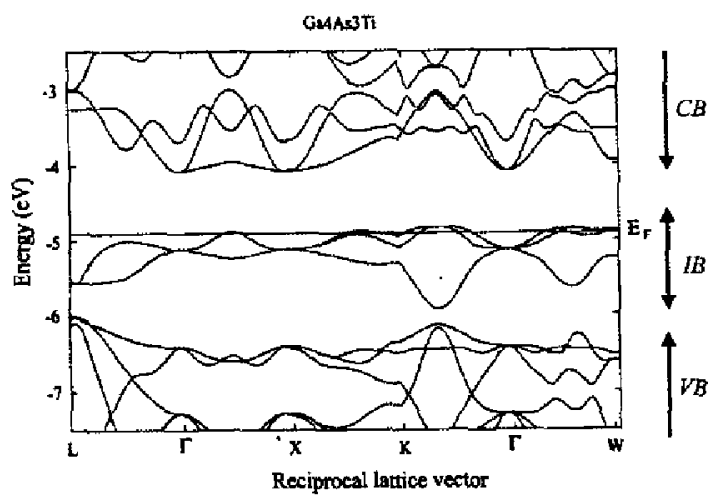

Fig. 7. Calculated bandgap diagram of a GaAsTi compound with the $\mathrm{Ti}$ atoms selectively substituting some As atoms in the conventional GaAs lattice. The position of the Fermi level in equilibrium, $E_{F_{\mathrm{T}}}$ is also shown, revealing the "metallic" character of the IB.

Research on this issue will focus from now on the determination of the chemical stability of these compounds, the prediction of their electricai and optical properties and the determination of suitable mechanism for their synthesis. Further published details on this subject can be found in references [11] and [12]

\section{THE HIGHLY POROUS MATERIAL APPROACH}

This approach is similar to the quantum dot one described above and is being investigated at the HahnMeitner Institut. Quantum dots are prepared within the nano-cavities of a transparent large bandgap semiconductor using a suitable absorber material. Due to the ran- dom structure of this arrangement multiple light reflection and strong scattering are expected [13]. The aim of this approach is to investigate a low cost version of the IBSC
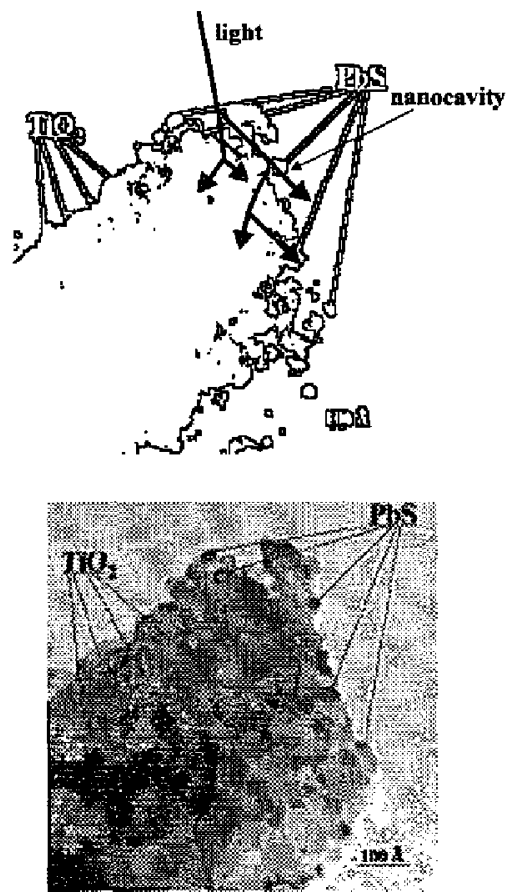

Fig. 8. Hlustration (above) of the formation of the nanocavities in a highly porous material and related lighttrapping in the material. Real (below) SEM picture.

One of the most interesting systems within this approach results to be that in which $\mathrm{TiO} 2$ and $\mathrm{PbS}$ constitute the porous and the absorber material, respectively. Fig. 8, (below) illustrates the QD-PbS formation. The corresponding band diagram has been determined in previous work [14] and is shown in Fig. 9. The average bandgap in the $\mathrm{PbS}$ quantum dots is approximately $1.85 \mathrm{eV}$, which is considerably larger than the $0.41 \mathrm{eV}$ bulk value for $\mathrm{PbS}$. The number of confined energy states in the valence band of the PbS will depend on the size of the dots and to what extent the dots are properly surrounded in the three spatial directions by a material that, like $\mathrm{TiO}_{2}$, produces a proper energy barrier at the $\mathrm{PbS}$ valence band. The $\mathrm{TiO}_{2}$ also provides the contact to the active part of the cell.

The $\mathrm{TiO}_{2}-(\mathrm{QD}-\mathrm{PbS})$ system has been used to look for experimental evidence of the two-photon up-converting mechanism that is implicit in the operation of the IBSC. To this end, samples of $\mathrm{TiO}_{2}$-(QD-PbS) have been immersed in a solution of methanol and $\mathrm{LiCl} / \mathrm{H} 2 \mathrm{O}$, and illuminated with photons of energy lower than the $\mathrm{TiO}_{2}$ bandgap $(3.2 \mathrm{eV})$. In the 2-photon up-converting mechanism some of these photons will excite electrons from the $\mathrm{TiO}_{2}$ valence band to some mid-bandgap states. These states 
correspond to interface states existing at the $\mathrm{TiO}_{2}-(\mathrm{QD}$ PbS) interface or to empty states of the valence band of the QD-PbS, whose wavefunction-tail penetrates into the $\mathrm{TiO}_{2}$. A second photon will then pump electrons from the QD-PbS valence band to its conduction band, and subsequent charge transfer will bring the excited electron to the $\mathrm{JiO}_{2}$ conduction band. The second excitation process, including the $\mathrm{PbS}$ excitation and charge transfer to the $\mathrm{TiO}_{2}$ conduction band, has been studied in some detail [\%4] and is known to have a large quantum efficiency. To detect the production of holes in the $\mathrm{TiO}_{2}$ valence band, a chemical process with high-energy selectivity was used: the oxidation of methanol which produces formaldehyde as a reaction product. This mechanism is labeled (4) in Fig. 9. It is well known that this process requires the high energy of $\mathrm{TiO}_{2}$ valence band holes. Since the process is in competition with recombination in the $\mathrm{TiO}_{2}$, labeled (3), the efficiency is expected to be low. Nonetheless, the formation of formaldehyde could recently be detected experimentally, a lower limit for the quantum efficiency of the initial step in the up-conversion mechanism could be obtained, and the existence of the complete process be derived.

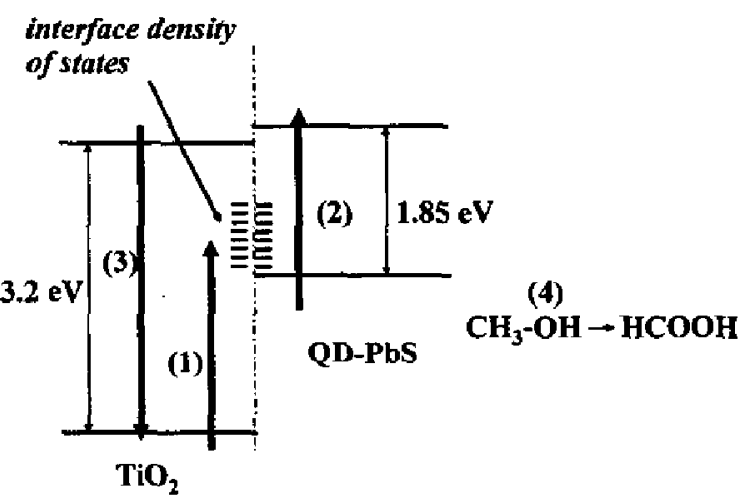

Fig. 9. Iliustration of the bandgap diagram of the porous $\mathrm{TiO}_{2}$-(QD-PbS) system. Arrows (1) and (2) represent photon absorption processes. (3) labels a recombination mechanism from the $\mathrm{TiO}_{2}$ conduction band to the valence band. (4) schematically illustrates the formation of a formaldehyde molecule from methanol.

\section{CONCLUSIONS}

Three approaches have been illustrated to implement in practice the IBSC concept: (a) engineering the structure by means of QD technology (b) direct synthesis of the intermediate band material and (c) use of highly porous materials. Among them, strategy (a) seems to be the most promising to fully demonstrate experimentally the physical principles involved in the operation of the JBSC. Strategy (b) has pointed towards specifically engineered photovoltaic candidate materials exhibiting half-filled intermediate band structure. Strategy (c) has allowed obtaining experimental evidence of the existence of a 2-photon up converting mechanism in a nano-porous structured material.

\section{ACKNOWLEDGMENTS}

Authors would like to acknowledge Dr. Richard Landford (University of Oxford) and Dr. Michael Giersig (HMi) for their work obtaining the TEM and SEM pictures of the QD samples. This work has been supported by the European Commission under contract ENK6 CT200 00310. The Instituto de Energia solar would like to acknowledge also the support of the Plan Naçional de I+D program under contract TiC2000-139). L. Cuadra is indebted to the Comunidad de Madrid for its financial support.

\section{REFERENCES}

[1] G.LAraújo and AMarti, "Absolute Limiting Efficiencies for Photo voltaic Energy Conversion", Solar Energy Materials and Solar Cells, 33, 1994, pp. 213-240.

[2] A.Luque and A.Marti, "Increasing the Efficiency of Ideal Solar Cells by Photon Induced Transitions at Intermediate Levels" Physcal Review Letters, 7B, N\$26, 1997, pp. 5014-5017.

[3] A.Luque and AMarti, "A Metallic Intermediate Band High Efficiency Solar Cell", Prog. in Phatov, Research and Applications, 9. N2, 2001,pp. 73-86.

[4] A.Luque, A.Marti and LCuadra, "High Efficiency Solar Cell with Metalic Intermediate Band", Proc. of the 16th European Photowot taic Solar Energy Conference, James \& James Ltd. London, 2000, pp. 5961.

[5] L.Cuadra, AMarti and A.Luque, "Modeling of the Absoption Coefficient of the Intermediate Band Solar Cell, Ptoc 16th European Photovoltaic Solar Energy Conference, James \& James Itd. London, 2000, pp. 15-21.

[6] M. Sugavara, "Seff-Assembled inGaAs'GaAs Quantum Dots", Academic Press, London, 1999.

[7] A.Marti, LCuadra and A.Luque, "Quantsm Dot intermediate Band Solar Cerr, Proc 28th Photovoltaics Specialist Conference, IEEE New York, 2000, p. 940-943.

[8] A.Martí, L.Cuadra and A.Luque, "Partal Filing of a Quantum Dot Intermediate Band for Solar Cells", IEEE Trans on Elec. Dev. 48, N. 10, 2001, pp. $2394-2399$.

[9] A.Marti, LCuadra and A.tuque, "Analysis of the space charge region of the quantum dot intermediate band solar cell Photovot taics for the 21st Century, Proc. 199th Electrochemical Society Meeting, The Eledrochernical Society, Pennington, 2001, pp. 46-60. [10] A.Martí, LCuadra and A.Luque "Design Constrains of the Quantum Dot intermediate Band Solar Cel", Intemational Workshop on Nanostuctures in Photovoltaics, Dresden, 2001 (To be published in Physica-E).

[11] C.Tablero, P.Wathon, LCuadra, AMarti, J.Femández, A.Luque, "Efficiencies of Halt-filed Intermediate Band Solar Cell Designed by First Principles Calculations" To be published in the Proc. of the 17th European Photovoltaic Solar Energy Conference (2002).

[12] P.Wahnon and C.Tablero, "Ab-lnitio electronic Stucture Catcutations for Metallic intermediate Band Fomation in Photowoltaic Materials", Phys. Rev. B, 65, 165115 (2002).

[13] Rolf R. Könenkamp, L Dloczik, K. Emst, and C. Olesch "Na nostructures for solar cells with externely thin absorbers", intema bional Workstop on Nanostructures in Photovoltaics, Dresden, 2001 (To be putlished in Physica-E)

[14] P. Hoyer and R. Könenkamp, "Photoconduction in porous TiOlsub 2/ sensilized by PbS quantum dots", Appl. Phys. Lelt 66,N.3p. 349-351 (1995). 